Supporting Information for

\title{
Excited State Dynamics in Dual-Defects Modified Graphitic Carbon Nitride
}

\author{
Shriya Gumber, ${ }^{1}$ Sraddha Agrawal, ${ }^{1}$ Oleg V. Prezhdo, ${ }^{1,2, *}$ \\ ${ }^{1}$ Department of Chemistry, University of Southern California, Los Angeles, CA 90089, USA \\ ${ }^{2}$ Department of Physics and Astronomy, University of Southern California, Los Angeles, CA 90089, \\ USA
}

*Corresponding author. E-mail: prezhdo@usc.edu

(a)

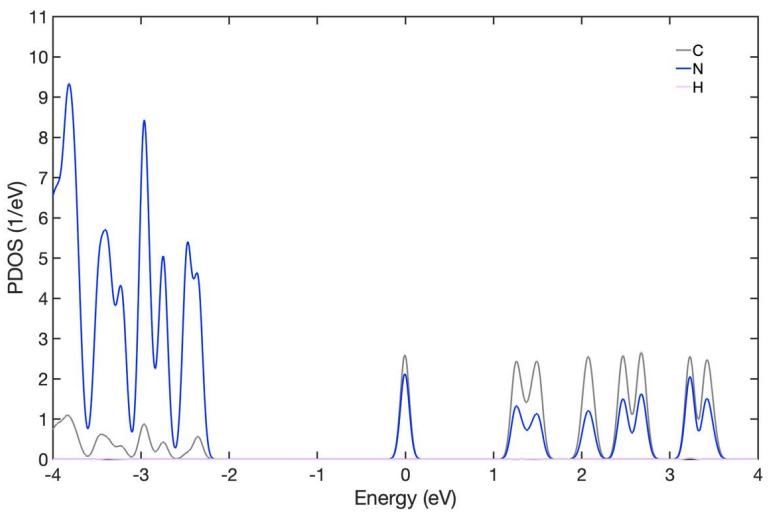

(b)

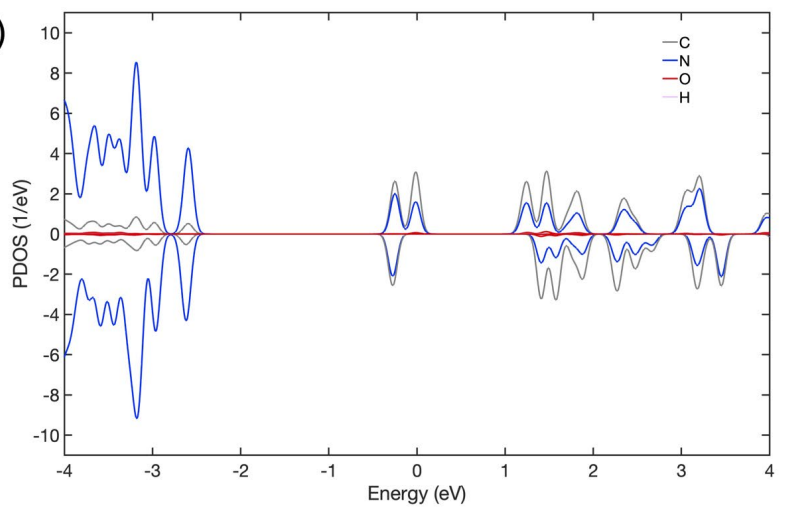

Figure S1. Projected density of states (PDOS) separated into contributions from atomic orbitals for (a) Ngen, and (b) ON-gen, obtained using hybrid HSE06 functional. The Fermi energy level is set to 0. 


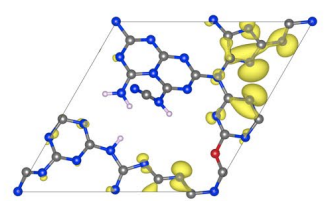

VBM

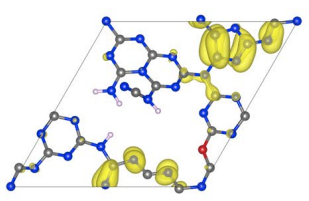

d1

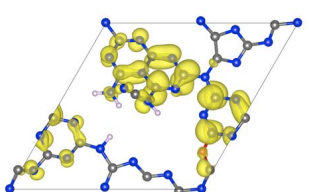

d2

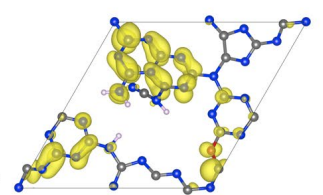

d3

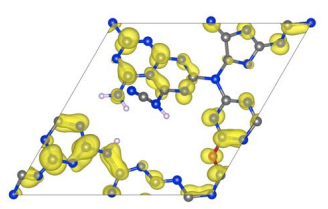

CBM

Figure S2. Charge densities of the KS orbitals for ON-gen for spin-down configuration. VBM represents valence band maximum and CBM represents conduction band minimum. Defect states are referred to as d1, d2 and d3, see Figure S4.

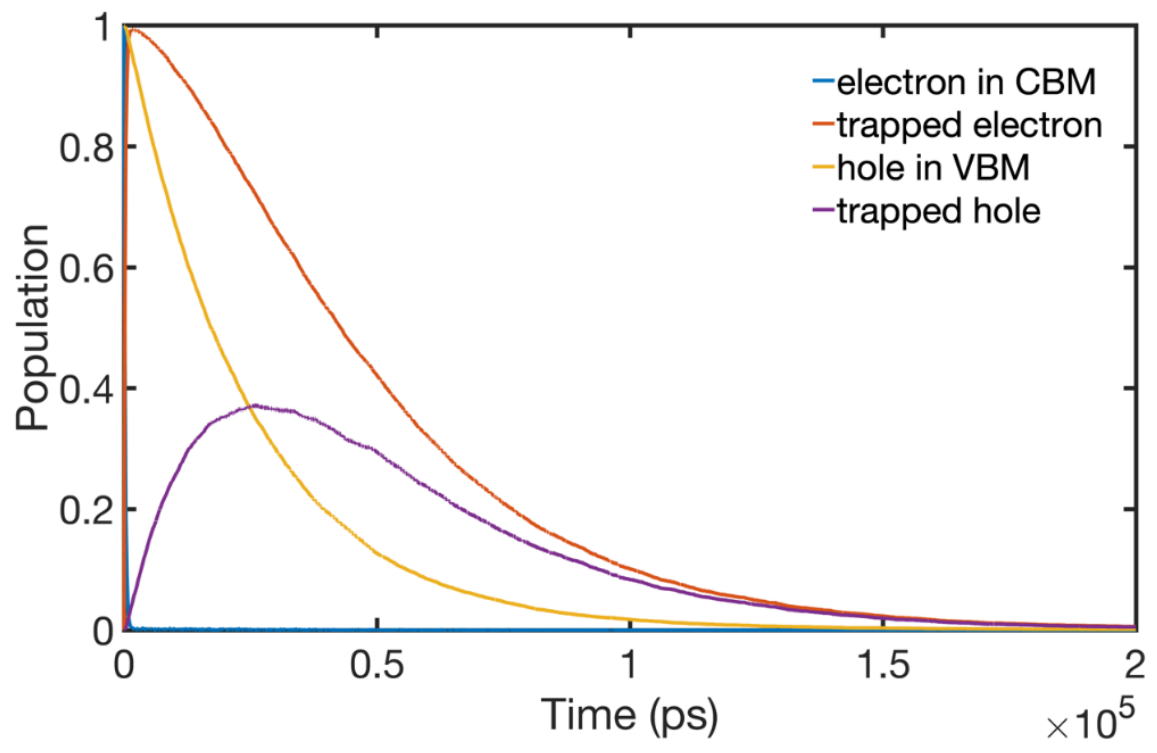

Figure S3. Non-radiative recombination dynamics in $\mathrm{ON}-g \mathrm{cn}$ for spin-down configuration. The corresponding spin-up channel data are shown in Figure $4 \mathrm{~b}$. 
(a)

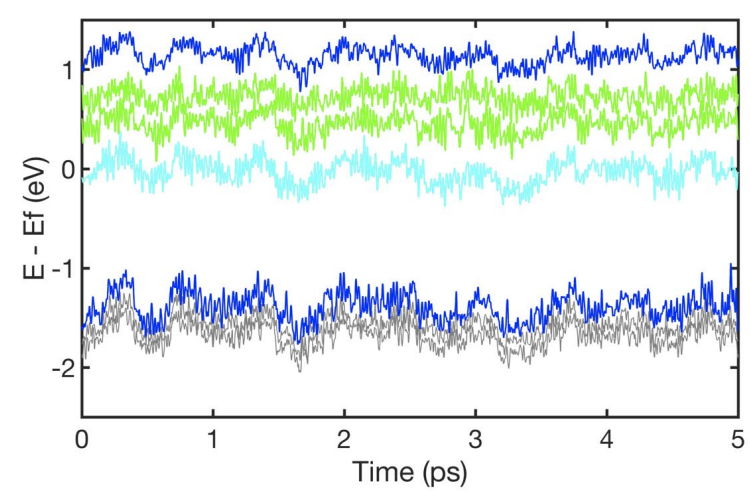

(b)

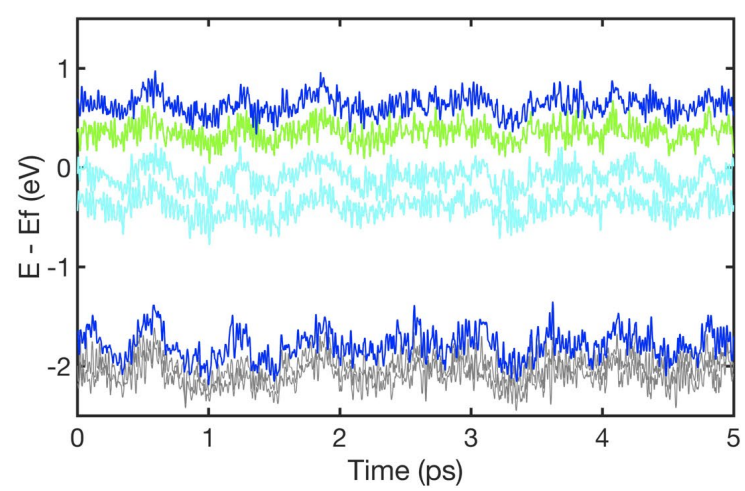

(c)

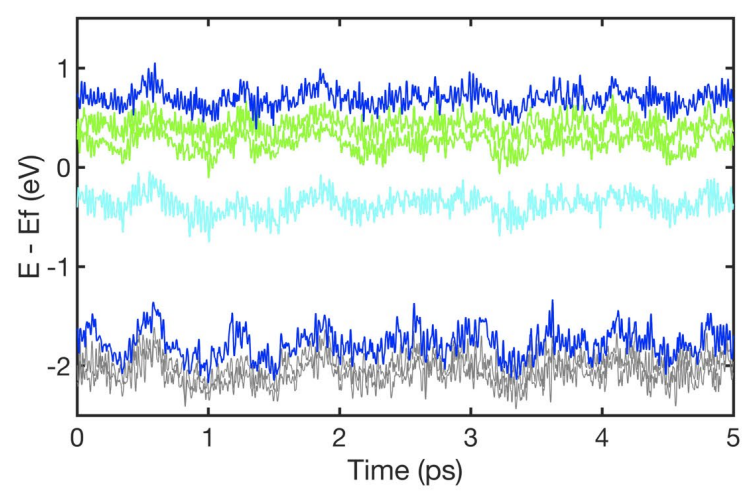

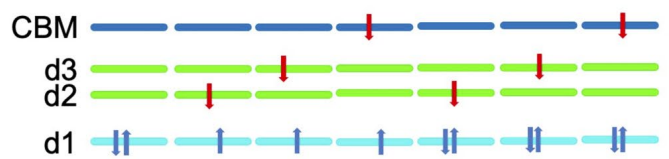
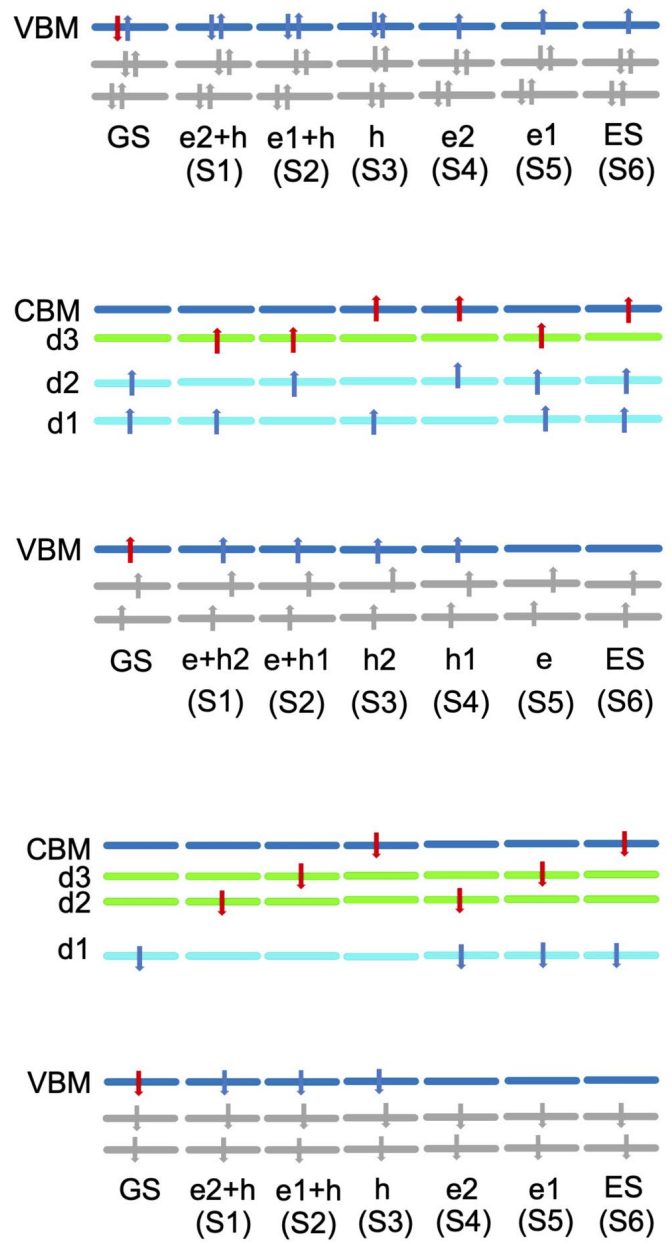

Figure S4. Evolution of the key KS levels and schematic of the active space configurations for chargecarrier trapping and recombination in (a) N-gen, (b) ON-gen (spin-up configuration), and (c) ON-gen (spin-down configuration). Blue represents the band-edges, cyan is for occupied defect states, and green is for vacant defect states. Gray is not a part of the NA-MD active space. The photoexcited electron is represented in red colour. Abbreviations used: gs- ground state; es- excited state; h- hole trap, e- electron trap. 
(a)

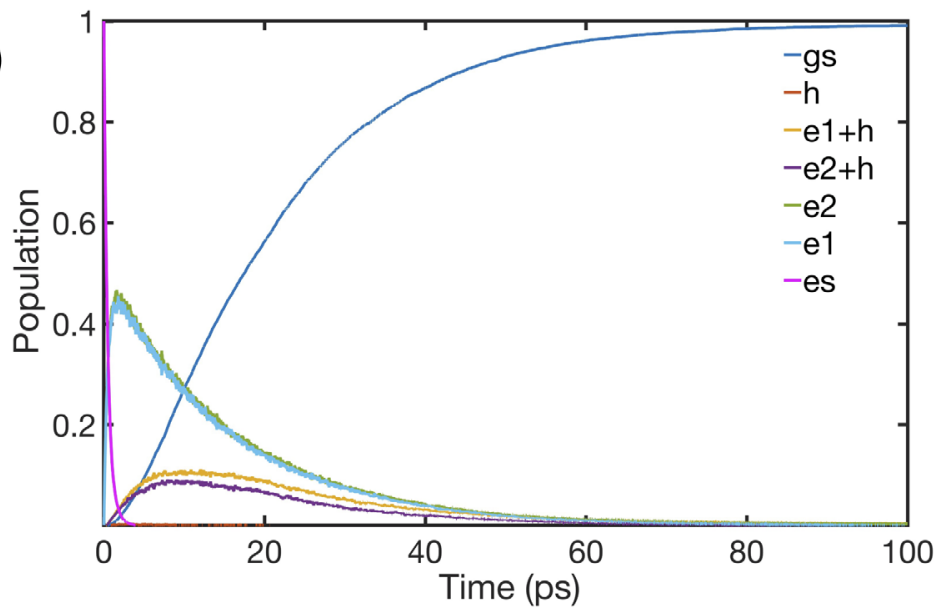

(b)

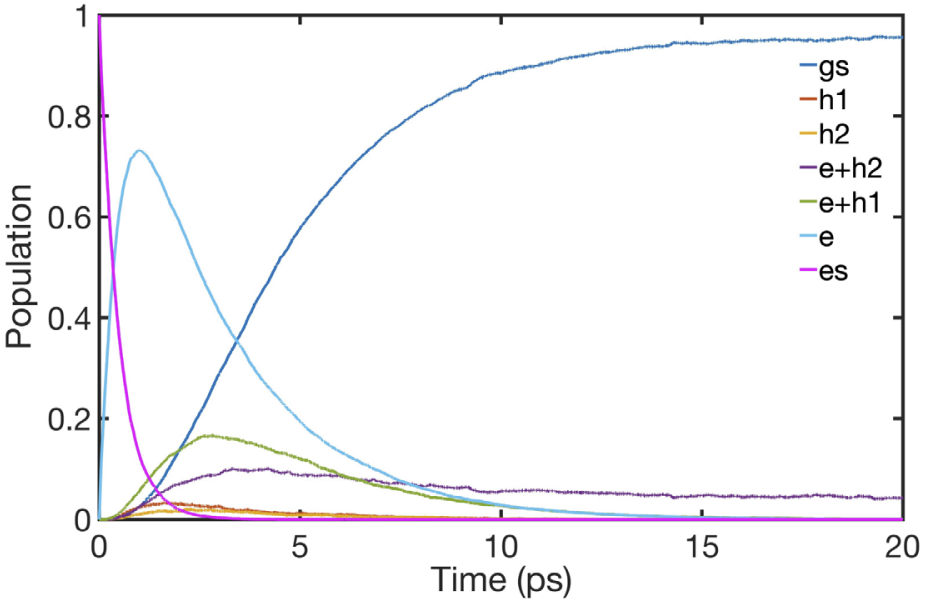

(c)

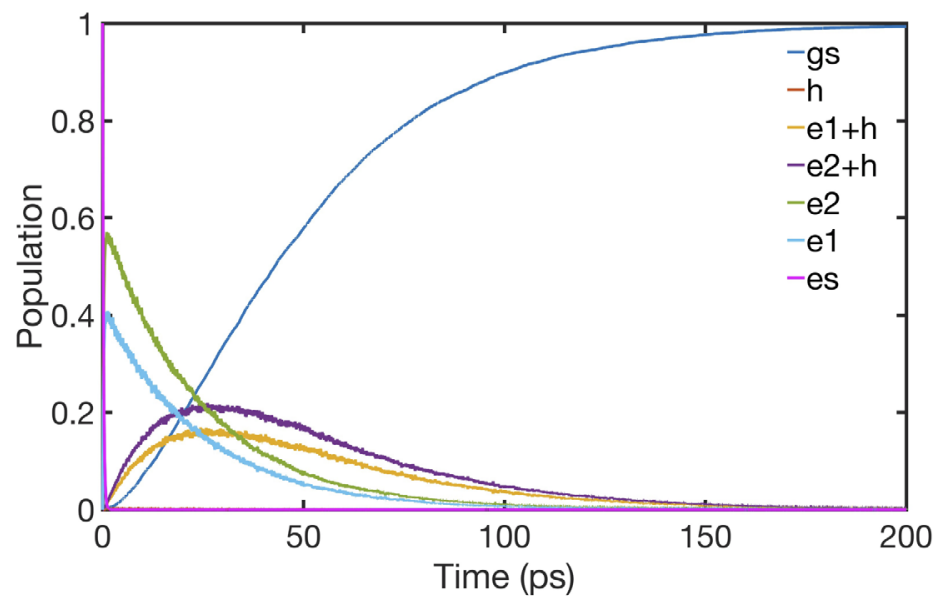

Figure S5. Detailed non-radiative trapping and recombination dynamics in (a) N-gcn, (b) ON-gen (spinup configuration), and (c) ON-gen (spin-down configuration). The configurations are described in Figure S4. 
Table S1. Time taken for the rise and decay of population of states in the NA-MD active space in picoseconds. The states are defined in Figure S4.

\begin{tabular}{ccccccccccccc}
\hline & GS & \multicolumn{2}{c}{$\mathrm{S} 1$} & \multicolumn{2}{c}{$\mathrm{S} 2$} & \multicolumn{2}{c}{$\mathrm{S} 3$} & \multicolumn{2}{c}{$\mathrm{S} 4$} & \multicolumn{2}{c}{$\mathrm{S} 5$} & $\mathrm{~S} 6$ \\
\hline & rise & rise & decay & rise & decay & rise & decay & rise & decay & rise & decay & decay \\
\hline N-gcn & 22.4 & 3.6 & 19.8 & 3.7 & 21.3 & 2.1 & 16.5 & 0.4 & 15.8 & 0.4 & 15.5 & 0.5 \\
& & & & & & & & & & & & \\
\hline $\begin{array}{c}\text { ON-gcn } \\
\text { spin up }\end{array}$ & $4.4(93 \%)$ & 1.8 & $64.5(53 \%)$ & 1.5 & 3.8 & 1.3 & 3.3 & 0.9 & 3.1 & 0.3 & 2.9 & 0.5 \\
\hline $\begin{array}{c}\text { ON-gen } \\
\text { spin down }\end{array}$ & 52.9 & 9.1 & 47.1 & 9.5 & 48.2 & 2.4 & 26.0 & 0.2 & 24.4 & 0.2 & 24.3 & 0.2 \\
\hline
\end{tabular}

